Diederik G. P. J. Geboers

Friso M. de Beer

Anita M. Tuip-de Boer

Tom van der Poll

Janneke Horn

Olaf L. Cremer

Marc J. M. Bonten

David S. Y. Ong

Marcus J. Schultz

Lieuwe D. J. Bos

\title{
Plasma suPAR as a prognostic biological marker for ICU mortality in ARDS patients
}

A. M. T. Boer

Laboratory of Experimental Intensive Care and Anesthesiology (LEICA), Academic

Medical Center, Amsterdam, The Netherlands

\section{T. van der Poll}

Center of Experimental and Molecular Medicine (CEMM), Academic Medical Center, Amsterdam, The Netherlands (Department of Intensive Care Medicine, University Medical Center Utrecht, Utrecht, the Netherlands); Jos F. Frencken, M.D. (Department of Intensive Care Medicine and Julius Center for Health Sciences and Primary Care, University Medical Center Utrecht, Utrecht, the Netherlands); and Roosmarijn T. M. van Hooijdonk, M.D., Mischa A. Huson, M.D., Laura R. A. Schouten, M.D., Marleen Straat, M.D., Lonneke A. van Vught, M.D., Maryse A. Wiewel, M.D., Esther Witteveen, M.D., Gerie J. Glas, M.D., and Luuk Wieske, M.D. (Department of Intensive Care Medicine, Academic Medical Center, University of Amsterdam).

Electronic supplementary material The online version of this article (doi:10.1007/s00134-015-3924-9) contains supplementary material, which is available to authorized users.

D. G. P. J. Geboers ( $)$ F. M. de Beer • J. Horn - M. J. Schultz - L. D. J. Bos Department of Intensive Care, Academic Medical Center, Meibergdreef 9, G3-228, 1105 AZ Amsterdam, The Netherlands e-mail: diederikgeboers@gmail.com

L. D. J. Bos

Department of Respiratory Medicine, Academic Medical Center, Amsterdam, The Netherlands

\section{O. L. Cremer · D. S. Y. Ong}

Department of Intensive Care Medicine, University Medical Center Utrecht, Utrecht, The Netherlands

M. J. M. Bonten · D. S. Y. Ong Department of Medical Microbiology, University Medical Center Utrecht, Utrecht, The Netherlands

M. J. M. Bonten - D. S. Y. Ong Julius Center for Health Sciences and Primary Care, University Medical Center Utrecht, Utrecht, The Netherlands
Abstract Purpose: We investi- gated the prognostic value of plasma soluble urokinase plasminogen activa- tor receptor (suPAR) on day 1 in patients with the acute respiratory dis- tress syndrome (ARDS) for intensive care unit (ICU) mortality and compared it with established disease severity scores on day 1. Methods: suPAR was determined batchwise in plasma obtained within $24 \mathrm{~h}$ after admission. Results: 632 ARDS patients were included. Significantly $(P=0.02)$ higher median levels of suPAR were

found with increasing severity of ARDS: $5.9 \mathrm{ng} / \mathrm{ml}$ [IQR 3.1-12.8] in mild ARDS $(n=82), 8.4 \mathrm{ng} / \mathrm{ml}$ [IQR 4.1-15.0] in moderate ARDS $(n=333)$, and $9.0 \mathrm{ng} / \mathrm{ml}[\mathrm{IQR}$ 4.5-16.0] in severe ARDS $(n=217)$. Non-survivors had higher median levels of suPAR [12.5 ng/ml (IQR 5.1-19.5) vs. $7.4 \mathrm{ng} / \mathrm{ml}$ (3.9-13.6), $P<0.001]$. The area under the receiver operator characteristic curve (ROCAUC) for mortality of suPAR (0.62) was lower than the ROC-AUC of the APACHE IV score $(0.72, P=0.007)$, higher than that of the ARDS definition classification $(0.53, P=0.005)$, and did not differ from that of the SOFA score $(0.68, P=0.07)$ and the oxygenation index $(\mathrm{OI})(0.58, P=0.29)$. Plasma suPAR did not improve the discrimination of the established disease severity scores, but did improve net reclassification of the APACHE score (29\%), SOFA score (23\%), OI (38\%), and Berlin definition classification (39\%). Conclusion: As a single biological marker, the prognostic value for death of plasma suPAR in ARDS patients is low. Plasma suPAR, however, improves the net reclassification, suggesting a potential role for suPAR in ICU mortality prediction models.

Keywords ARDS - ICU mortality · suPAR - Biological marker 


\section{Introduction}

Mortality rates of patients with the acute respiratory distress syndrome (ARDS) are high [1, 2]. Biological markers as well as disease severity scoring systems may be useful for risk stratification and the creation of homogenous patient groups in clinical trials [3]. However, currently no single biological marker has been found to have sufficient discriminative value for accurate prediction of mortality or bedside decision making in ARDS patients in the clinical setting, although thrombomodulin has very recently been described as a promising marker [4].

Soluble urokinase plasminogen activator receptor (suPAR) derives from proteolytic cleavage of the urokinase plasminogen activator receptor from the cell membrane and is thought to reflect activation of the immune system [5]. suPAR is an emerging biomarker in sepsis research. Previous studies in critically ill patients with sepsis or bacteremia have shown a strong association between increasing levels of plasma suPAR and mortality [6-8].

We hypothesized plasma suPAR levels on admission to have prognostic value in intensive care unit (ICU) patients with ARDS. The aim of this study was to determine the prognostic value of plasma suPAR on admission for ICU mortality and to compare it with existing severity scoring systems. Importantly, we also investigated the additive value (net reclassification improvement) of the biomarker on top of existing scoring systems [9]. In addition, we determined the prognostic value of plasma suPAR on admission for duration of ventilation and length of stay in ICU.

\section{Methods}

\section{Study design}

This was a prospective cohort study as part of the 'Molecular Diagnosis and Risk Stratification' (MARS) study performed in the ICUs of two tertiary care hospitals in the Netherlands, the Academic Medical Center in Amsterdam and the University Medical Center in Utrecht [10]. The medical ethics committees of both hospitals approved the study protocol and the opt-out consent method. The patient or their legal representative was presented with a brochure and opt-out form, to be completed in case of unwillingness to participate.

\section{Setting}

Both ICUs are closed-format units, in which a team of board-certified critical care physicians, fellows in critical care medicine, and board-certified ICU nurses care for a mixed medical-surgical population of patients. The nurse to patient ratio was $1: 1-1: 2$, depending on disease severity. Patients received lung-protective mechanical ventilation with lower tidal volumes, higher levels of positive end-expiratory pressure levels and prone ventilation if necessary (i.e., in case of severe refractory hypoxemia) [11]. Furthermore, a restrictive fluid strategy was followed [12], and analgo-sedation was performed using sedation scales with bolus sedation [13] or continuous sedation.

\section{Inclusion and exclusion criteria}

Consecutive adult patients admitted to the ICU with an expected length of stay of more than $24 \mathrm{~h}$ from January 2011 to December 2013 were eligible for participation in the MARS study. Patients enrolled between January 2011 and June 2013, with ARDS and with available $\mathrm{PaO}_{2} / \mathrm{FiO}_{2}$ on day 1 needed for classification of ARDS severity, were eligible for inclusion in the present analysis. Because the level of plasma suPAR was determined on day 1, patients that did not fulfill the criteria for ARDS upon admission were excluded. ARDS was defined according to the criteria stated by the American-European Consensus Conference (AECC) on ARDS [14]: i.e., the diagnosis required an acute onset of symptoms, the presence of bilateral infiltrates on chest radiography, a pulmonaryartery wedge pressure below $18 \mathrm{mmHg}$ and/or the absence of signs of left ventricular dysfunction, and a partial pressure of oxygen in arterial blood to fraction of inspired oxygen ratio $(\mathrm{P} / \mathrm{F})$ no greater than 200 . Patients that met the above criteria but with a $\mathrm{P} / \mathrm{F}$ ratio of 300 or below, classified as ALI according to AECC criteria, were also included in the analysis because the updated definition of ARDS according to the Berlin definition $[15,16]$ classifies this category as mild ARDS. Henceforth in this article patients with ALI according to AECC criteria will be categorized as mild ARDS. Although our study started in 2011, before the recent Berlin update of the ARDS definition was published [15], we found that $0 \%$ of the study cohort would have been excluded had we not used the new definitions. There were no additional inclusion or exclusion criteria.

\section{Diagnosis of ARDS and its cause}

ARDS was diagnosed by a dedicated team of researchers who were trained in the proper use of the criteria stated by the AECC on ARDS [14]. Patients were classified as having mild, moderate, or severe ARDS using the lowest $\mathrm{PaO}_{2} / \mathrm{FiO}_{2}$ on day 1 . The cause for ARDS was determined and scored in the following categories: pneumonia, aspiration, other pulmonary (i.e., inhalation trauma, near 
drowning), sepsis, trauma or major surgery, pancreatitis or other non-pulmonary cause (i.e., blood transfusion, toxic medication). In the event of multiple causes for ARDS, each cause was scored separately. The predetermined subgroup with indirect causes for ARDS contained all patients in which direct causes (i.e., pneumonia, aspiration, or other pulmonary) were absent. A patient was called a smoker when smoking actively prior to admission or a drinker when drinking more than 4 units of alcohol per day. Diabetes mellitus encompassed patients using insulin or oral antidiabetic medication prior to admission. Immune deficiency was defined as current use of immunosuppressive drugs, chemo- or radiotherapy in the previous 12 months, or documented humoral or cellular deficiency. Cardiovascular disease encompassed patients with chronic heart failure (i.e., NYHA classification 2-4), prior myocardial infarction, or signs of chronic central or peripheral vascular disease (i.e., intermittent claudication, history of percutaneous transluminal angioplasty or bypass surgery, thoracic or abdominal aneurysm larger than $6 \mathrm{~cm}$, history of transient ischemic attack or cerebrovascular accident). Malignancy was defined as a history of malignant neoplasms, with or without metastases, verified by evident clinical or pathological tests or the following hematological malignancies: lymphoma, leukemia, or multiple myeloma.

\section{Established disease severity scoring systems}

The Acute Physiology and Chronic Health Evaluation (APACHE) IV score [17], the Sequential Organ Failure Assessment (SOFA) [18] score, the Oxygenation Index (OI) [19], and the Berlin classification [15, 16] were determined in the first $24 \mathrm{~h}$ after admission.

\section{Outcome}

The primary outcome, ICU mortality, was defined as the occurrence of death during ICU follow-up. Secondary outcomes included the number of ICU-free days, defined as the number of days alive and not admitted to the ICU at day 28 and day 90 , and the number of ventilator-free days, defined as the number of days alive and free from mechanical ventilation at day 28 and day 90 . In secondary analyses, 30- and 90-day mortality, defined as the occurrence of death at 30 or 90 days, were used as the outcome.

\section{Sample handling}

Blood samples were collected on admission to the ICU and were immediately frozen at $-80{ }^{\circ} \mathrm{C}$. Plasma suPAR levels were determined batchwise using the suPARnostic immunoassay kit (Virogates, Copenhagen, Denmark) according to manufacturer's instructions.

\section{Statistical analysis}

Data were presented as medians and interquartile ranges. Differences between ARDS severity groups were compared using Mann-Whitney $U$ test or Kruskal-Wallis ANOVA for differences between more than two groups. Correlations between variables were analyzed using Spearman's rank test. For regression analysis, data without normal distribution were logarithmically transformed.

Univariate logistic regression models were used to study and compare the association between plasma suPAR levels, the APACHE IV score, the SOFA score, the OI, and the Berlin definition on the first day of stay in ICU, and outcome. Calibration of the model was performed with a residual plot and a Hosmer and Lemeshow goodness-of-fit test. The discriminative capacities of the models were shown using the area under the curve of the receiver operating characteristic curve (ROC-AUC), with bootstrapped $95 \%$ confidence interval $(95 \%$ CI) [20]. The optimal cut-off was determined using a Youden index for the selection of the highest sum of sensitivity and specificity. The prognostic accuracy was assessed in several subgroups by comparing the ROC-AUC for mortality per severity classification of ARDS, septic vs. non-septic patients, direct vs. indirect ARDS, and medical vs. surgical patients. A sensitivity analysis was performed by comparing the odds ratios for the prediction of ICU mortality in an unadjusted logistic regression model to the odds ratio in a logistic regression model including a covariate. The prognostic value of suPAR was found to be sensitive to changes if the log odds ratio changed by more than 0.1 when the covariate was added. The following covariates were investigated: severity of ARDS (i.e., mild, moderate, or severe), cause of ARDS (i.e., direct or indirect, sepsis or non-sepsis), type of admission (i.e., acute or elective, and medical or surgical), comorbidities (diabetes mellitus, immune deficiency, cardiovascular disease, malignancy), smoking status (i.e., active smoker or none), alcohol consumption (i.e., active drinker or none), APACHE IV score, SOFA score on day 1 , the OI, and the Berlin definition.

Next, the association between suPAR and the secondary outcomes, ICU-free days and ventilator-free days at day 28 and day 90, was determined using univariate linear regression models.

Finally, the combined prognostic accuracy of suPAR and the APACHE IV score, SOFA score, OI, and Berlin classification was analyzed in a bivariable logistic regression model. Single marker predictions were compared to composite models using DeLongs test for differences in ROC-AUC and by means of net reclassification improvement (NRI) and integrated discrimination improvement (IDI) [9]. The NRI compares the accuracy 
of the models with and without suPAR by calculating the number of subjects whose risk is more accurately classified and the proportion of subjects whose risk is less accurately classified. By subtracting these values a net reclassification improvement is determined. The IDI also calculates reclassification, but in this case risk is stratified as a continuous variable.

SPSS software version 22.0 (IBM Corp., Armonk, NY, USA) and R [21] were used for statistical analysis. Functions not available in SPSS were computed with $\mathrm{R}$ using R-studio interface. A $P$ value less than 0.05 was used to determine statistical significance for all tests.

\section{Results}

Patient demographics and plasma suPAR levels

Of 7784 patients admitted to the ICU in 2011 and 2012, $702(9.0 \%)$ were diagnosed with ARDS on admission and could be classified as mild, moderate, or severe ARDS. A plasma suPAR level was available in 632 patients $(90.0 \%)$. All patients were mechanically ventilated. Patient characteristics are presented in Table 1 and causes for ARDS in Table S1 in the electronic supplementary material. The two most common causes for ARDS were sepsis and pneumonia. ICU mortality rate was $25.3 \%$, which was not different in the three severity groups $(P=0.22)$. Differences between patients who died in the ICU and those who survived till ICU discharge are presented in Table 1.

Higher plasma levels of suPAR were found with increasing severity of ARDS: $5.9 \mathrm{ng} / \mathrm{ml}$ [IQR 3.1-12.8] in mild ARDS $(n=82), 8.4 \mathrm{ng} / \mathrm{ml}$ [IQR 4.1-15.0] in moderate ARDS $(n=333)$, and $9.0 \mathrm{ng} / \mathrm{ml}$ [IQR 4.5-16.0] in severe ARDS $(n=217)$. Non-survivors had higher median levels of suPAR $[12.5 \mathrm{ng} / \mathrm{ml}$ (IQR 5.1-19.5) vs. $7.4 \mathrm{ng} / \mathrm{ml}$ (3.9-13.6), $P<0.001]$ (Fig. 1). Plasma levels of suPAR correlated with the number of ICU-free days at day 28 and $90(r=-0.24, p<0.001$ for both) and the number of ventilator-free days at day 28 and day $90 \quad(r=-0.23, p<0.001$ and $r=-0.22$, $p<0.001$, respectively), higher levels being found in patients who had less ICU- or ventilator-free days.

\section{Prediction of ICU mortality}

Increased levels of plasma suPAR were significantly associated with ICU mortality (Table 2). Calibration showed a poor model fit $\left(\chi^{2}=20.9, P=0.008\right)$ (Fig. S1 in the electronic supplementary material). The ROC-AUC was $0.62(95 \%$ CI $0.57-0.68)$ (Table 2). The Youden index revealed an optimal cut-off at a suPAR level of $11.7 \mathrm{ng} / \mathrm{ml}$. At this cut-off sensitivity is $54 \%$ and specificity $68 \%$, corresponding to a positive likelihood ratio of 1.69 and a negative likelihood ratio of 0.67 .

There were no differences in the association between suPAR and mortality when comparing plasma levels of suPAR stratified by severity of ARDS (i.e., mild, moderate, severe), by type of admission (i.e., medical and surgical), and between septic and non-septic patients. For patients with an indirect cause of ARDS, plasma suPAR showed a trend towards a prognostic value compared to patients with a direct cause of ARDS (see Table 3 for odds ratios and ROC-AUCs).

The odds ratio of suPAR was sensitive to changes in the APACHE IV score (OR 1.30) and the SOFA score (OR 1.36). It was not sensitive to changes in the OI (OR 1.52) or Berlin definition classification (OR 1.65), nor age (OR 1.68), comorbidities (OR 1.60), cause of ARDS (OR 1.75), type of admission (OR 1.63), smoking (OR 1.65), or alcohol consumption (OR 1.65).

Table 2 displays the predictive values of the Berlin classification, OI, SOFA, and APACHE IV for mortality. There were no significant differences in ROC-AUC between suPAR and the OI $(P=0.29)$ and the SOFA score $(P=0.07)$. Plasma suPAR outperformed the Berlin classification $(P=0.005)$, but the APACHE IV score $(P=0.007)$ was the best predictor of mortality (Table 2$)$.

Increased levels of suPAR were significantly associated with 30-day mortality and 90-day mortality: OR 1.36 (95\% CI 1.11-1.66) and OR 1.34 (95\% CI 1.12-1.61), respectively. The ROC-AUC was $0.58 \quad(95 \% \mathrm{CI}$ $0.52-0.63)$ for 30-day mortality and 0.58 (95\% CI $0.53-0.63$ ) for 90 -day mortality.

Combining suPAR with the existing disease severity scores

The ROC-AUC for the APACHE IV score $(P=0.22)$, SOFA score $(P=0.28)$, OI $(P=0.75)$, and Berlin classification $(P=0.98)$ did not improve when suPAR was added to the model (Table 2). The net reclassification improvement was 0.28 (or $28 \%, P=0.002$ ) and the IDI was $0.01(P=0.01)$ for the APACHE IV score (see Fig. 2 for the risk reclassification plot); the NRI was 0.23 (or $23 \%, P=0.01)$ and the IDI was $0.01(P=0.04)$ for the SOFA score; the NRI was 0.38 (or $38 \%, P<0.001$ ) and the IDI was $0.02(P=0.001)$ for the OI; the NRI was 0.39 (or $39 \%, P<0.001$ ) and the IDI was 0.03 $(P<0.001)$ for the Berlin classification.

\section{Secondary outcomes}

Plasma suPAR levels were predictive of the number of ICU-free days at day $28(\beta=-0.53, P<0.001$, adjusted $\left.R^{2}=0.04\right)$ and at day $90 \quad(\beta=-0.59, \quad P<0.001$, adjusted $\left.R^{2}=0.03\right)$. They were also predictive of the 
Table 1 Patient characteristics

\begin{tabular}{|c|c|c|c|c|}
\hline & Entire cohort & Survivors (472) & Non-survivors (160) & $P$ value \\
\hline Age (years) & $62(51-71)$ & $62(49-71)$ & $63(54-72)$ & 0.21 \\
\hline Male (yes) & $402(64)$ & $294(62)$ & $108(68)$ & 0.24 \\
\hline suPAR (ng/ml) & $8.4(4.1-15.1)$ & $7.4(3.9-13.6)$ & $12.5(5.1-19.5)$ & $<0.001$ \\
\hline APACHE IV & $83(65-104)$ & $78(61-99)$ & $100(82-124)$ & $<0.001$ \\
\hline SOFA & $8(6-11)$ & $8(6-10)$ & $10(8-13)$ & $<0.001$ \\
\hline OI & $7.4(4.9-10.9)$ & $7.0(4.7-10.1)$ & $7.8(5.7-13.0)$ & 0.005 \\
\hline ICU mortality & $160(25)$ & & & \\
\hline Length of stay (days) & $9(5-17)$ & $9(6-17)$ & $7(2-16)$ & $<0.001$ \\
\hline ICU-free days at day 28 (days) & $15(0-21)$ & $19(11-22)$ & 0 & $<0.001$ \\
\hline ICU-free days at day 90 (days) & $77(0-83)$ & $81(73-84)$ & 0 & $<0.001$ \\
\hline Ventilation (days) & $7(3-13)$ & $7(4-13)$ & $7(2-15)$ & 0.13 \\
\hline VFD at day 28 & $17(0-23)$ & $21(15-24)$ & 0 & $<0.001$ \\
\hline VFD at day 90 & $79(0-85)$ & $83(77-86)$ & 0 & $<0.001$ \\
\hline \multicolumn{5}{|c|}{ ARDS classification upon admission } \\
\hline Mild & $82(13)$ & $60(13)$ & $22(14)$ & 0.74 \\
\hline Moderate & $333(53)$ & $258(55)$ & $75(47)$ & 0.09 \\
\hline Severe & $217(34)$ & $154(33)$ & $63(39)$ & 0.12 \\
\hline \multicolumn{5}{|l|}{ Admission type } \\
\hline Medical & $460(73)$ & $337(71)$ & $123(77)$ & 0.18 \\
\hline Surgical elective & $70(11)$ & $58(12)$ & $12(8)$ & 0.10 \\
\hline Surgical emergency & $102(16)$ & $77(16)$ & $25(16)$ & 0.84 \\
\hline \multicolumn{5}{|l|}{ Co-morbidities } \\
\hline Diabetes & $105(17)$ & 79 (17) & $26(16)$ & 0.89 \\
\hline Immune deficiency & 117 (19) & 79 (17) & $38(24)$ & 0.05 \\
\hline Cardiovascular disease & $164(26)$ & $131(28)$ & $33(21)$ & 0.08 \\
\hline Malignancy & $128(20)$ & $85(18)$ & $43(27)$ & 0.02 \\
\hline Smoking (yes) & $71(11)$ & $60(13)$ & $11(7)$ & 0.04 \\
\hline Alcohol (yes) & $90(14)$ & $70(15)$ & $20(13)$ & 0.47 \\
\hline
\end{tabular}

Patient characteristics for the entire cohort and for the survivor and non-survivor subgroups are demonstrated. Continuous variables are expressed as median (25th-75th percentile). Categorical variables are expressed as number (percentage). Differences between groups were tested using Mann-Whitney $U$ or Chi-square test and $P$ values are reported

$A R D S$ acute respiratory distress syndrome, suPAR soluble urokinase plasminogen activator receptor, APACHE IV Acute Physiology and Chronic Health Evaluation IV, SOFA Sequential Organ Failure Assessment score, OI oxygenation index, length of stay in the ICU, Ventilation number of days of mechanical ventilation, $V F D$ ventilator-free days

number of ventilator-free days at day $28(\beta=-0.55$, $P<0.001$, adjusted $\left.R^{2}=0.04\right)$ and at day 90 $\left(\beta=-0.59, P<0.001\right.$, adjusted $\left.R^{2}=0.03\right)$.

\section{Discussion}

This was the first study that investigated the association between plasma suPAR and mortality in a cohort of ARDS patients. Plasma levels of suPAR were higher with increasing severity of ARDS, and higher in patients who died in the ICU. Furthermore, higher plasma suPAR levels were associated with less ventilator- and ICU-free days. While the prognostic value of suPAR as a single biological marker was low, combined it with existing disease severity scores suPAR improved the net reclassification.

The ROC-AUC for mortality of suPAR was comparable to previous investigations in ventilated critically ill patients, which could have included many patients with ARDS [6-8]. However, the ROC-AUC was low, which

could at least in part be because of the high mortality in these patients. In comparison, the plasma level of suPAR was found to have a good predictive value for mortality in patients with mild malaria, with a corresponding low mortality, but lost its predictive value in severe malaria, with a corresponding high mortality [22]. Notably, the predictive value of suPAR did not improve with less severe forms of ARDS (i.e., mild or moderate ARDS).

We found a trend towards a prognostic value of suPAR for patients with an indirect cause of ARDS, but not for patients with a direct cause of ARDS. A previous study in patients with inhalation trauma found higher levels of suPAR in bronchoalveolar lavage fluid of patients compared to controls, while systemic levels were similar in both groups [23]. This finding suggests local production of suPAR in the lung, which may cause plasma levels to remain relatively low in direct causes of ARDS. Consequently, the predictive value of plasma levels of suPAR could be lower in cases of direct ARDS as compared to indirect ARDS. Also we found that the association between plasma suPAR and mortality was independent of the occurrence of sepsis, i.e., the 
suPAR levels: survivors vs non-survivors \& per ARDS classification

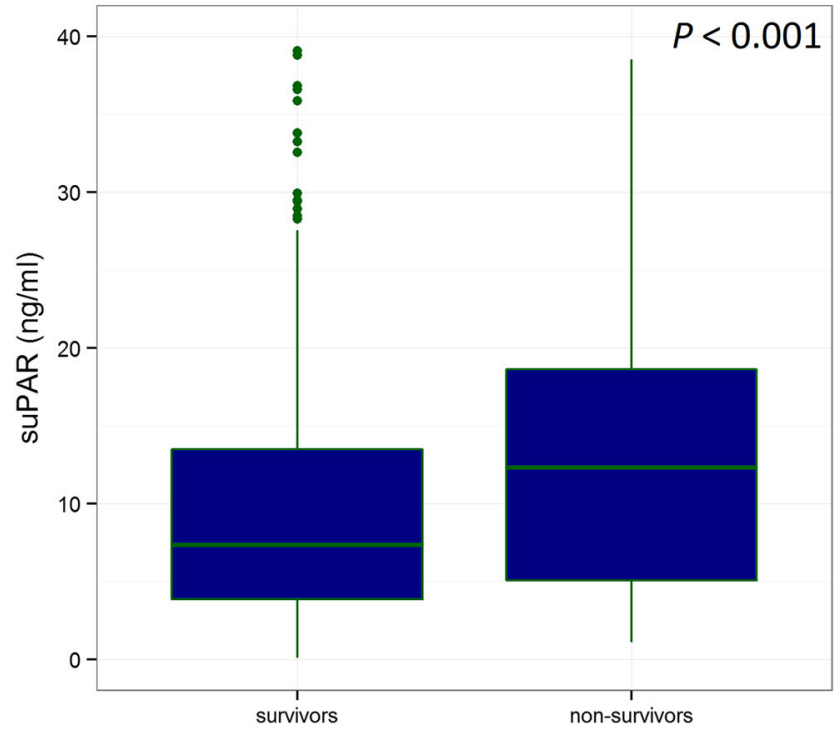

Fig. 1 Box plot showing the differences in suPAR levels for survivors/non-survivors $(P<0.001)$ and suPAR levels per ARDS classification. The differences in suPAR levels are significant when comparing all subgroups $(P=0.02)$. In post hoc Mann-Whitney

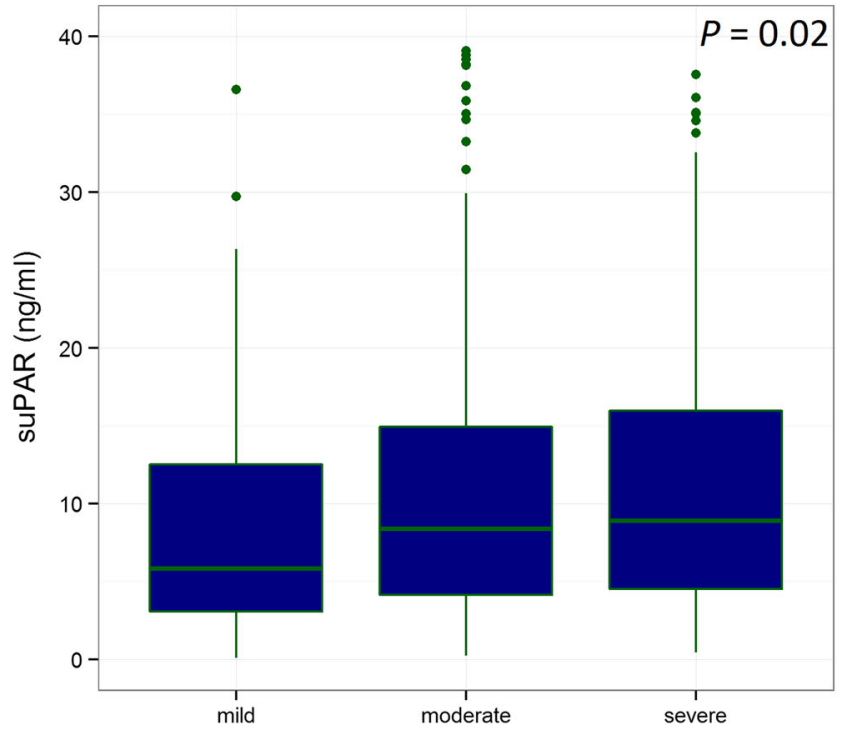

$U$ analysis suPAR levels for patients with mild ARDS differed from those with moderate $(P=0.03)$ and severe ARDS $(P=0.004)$. There was no difference in suPAR levels for patients with moderate and severe ARDS $(P=0.21)$

Table 2 Outcomes logistic regression models of all predictors of mortality and ROC-AUCs for combined models

\begin{tabular}{|c|c|c|c|c|c|}
\hline & OR for ICU mortality & $95 \% \mathrm{CI}$ & $P$ value & ROC-AUC & $95 \%$ CI \\
\hline suPAR & 1.65 & $1.32-2.05$ & $<0.001$ & 0.62 & $0.57-0.68$ \\
\hline APACHE IV & 13.59 & $7.05-26.21$ & $<0.001$ & 0.72 & $0.68-0.76$ \\
\hline SOFA & 1.20 & $1.14-1.27$ & $<0.001$ & 0.68 & $0.63-0.74$ \\
\hline OI & 1.76 & $1.28-2.42$ & 0.001 & 0.58 & $0.52-0.64$ \\
\hline Berlin classification & 1.14 & $0.87-1.51$ & 0.34 & 0.53 & $0.47-0.58$ \\
\hline SUPAR + APACHE IV & & & & 0.73 & $0.68-0.77$ \\
\hline SuPAR + SOFA & & & & 0.69 & $0.63-0.74$ \\
\hline suPAR + OI & & & & 0.64 & $0.58-0.69$ \\
\hline suPAR + Berlin class & & & & 0.62 & $0.57-0.68$ \\
\hline
\end{tabular}

A one point increase in plasma suPAR is associated with a $1.65-$ fold increase in ICU mortality. Similarly a one point increase in APACHE IV score, SOFA score, and OI is associated with a 13.59 , 1.20 , and 1.76 increase in mortality, respectively. Upscaling from one stage of the Berlin classification to another would mean a 1.14fold mortality increase

association was found in both septic and non-septic ARDS patients, suggesting that suPAR is not solely a component of the sepsis pathway.

Our findings demonstrate that the prognostic value of suPAR plasma levels is not strong enough for it to function as a singular biomarker for mortality in ARDS patients, but its addition to other biomarkers or prediction scores might be clinically relevant. The APACHE IV score had better discriminative capacity than SuPAR, yet this does not necessarily impede the use of plasma suPAR in a clinical setting. Even though this scoring system has proven useful in the prediction of mortality for groups of
OR odds ratio, SUPAR soluble urokinase plasminogen activator receptor, APACHE IV Acute Physiology and Chronic Health Evaluation IV, SOFA Sequential Organ Failure Assessment score, $O I$ oxygenation index

critically ill patients, it is not developed for risk stratification in individual patients for inclusion in clinical trials or treatment decisions [24]. Furthermore its clinical use is limited because collection of necessary data is time consuming, expensive, and the results are prone to interobserver variability $[24,25]$. In contrast, suPAR is a feasible biological marker for clinical use, with limited circadian changes in plasma levels, stability to freezethaw procedures and reliable measurement by a simple ELISA [5].

Owing to its feasible use in clinical practice suPAR could be a biological marker to combine with other 
Table 3 Subgroup analyses

\begin{tabular}{|c|c|c|c|c|}
\hline & OR for ICU mortality $(95 \% \mathrm{CI})$ & $P$ value & ROC-AUC (95 \% CI) & $P$ value for DeLongs test \\
\hline \multicolumn{5}{|c|}{ ARDS severity classification } \\
\hline Mild & $1.47(0.85-2.54)$ & 0.17 & $0.59(0.44-0.74)$ & \multirow[t]{3}{*}{0.89} \\
\hline Moderate & $1.66(1.20-2.28)$ & 0.002 & $0.63(0.55-0.70)$ & \\
\hline Severe & $1.73(1.19-2.52)$ & 0.004 & $0.64(0.58-0.69)$ & \\
\hline \multicolumn{5}{|c|}{ Cause ARDS } \\
\hline Direct & $1.36(1.02-1.83)$ & 0.04 & $0.58(0.51-0.65)$ & \multirow[t]{2}{*}{0.06} \\
\hline Indirect & $2.12(1.51-2.99)$ & $<0.001$ & $0.69(0.61-0.76)$ & \\
\hline \multicolumn{5}{|l|}{ Sepsis } \\
\hline Yes & $1.69(1.26-2.28)$ & 0.001 & $0.63(0.57-0.70)$ & \multirow{2}{*}{0.63} \\
\hline No & $1.62(1.13-2.33)$ & 0.008 & $0.61(0.52-0.70)$ & \\
\hline \multicolumn{5}{|c|}{ Type of admission } \\
\hline Medical & $1.66(1.28-2.15)$ & $<0.001$ & $0.63(0.57-0.69)$ & \multirow[t]{2}{*}{0.56} \\
\hline Surgical & $1.56(0.98-2.49)$ & 0.06 & $0.60(0.49-0.70)$ & \\
\hline
\end{tabular}

The association between plasma levels of suPAR for ICU mortality was further stratified by severity of ARDS, i.e., mild, moderate or severe ARDS, cause of ARDS, i.e., direct or indirect, presence of sepsis or type of admission, i.e., medical or surgical. For each subcategory a ROC-AUC was calculated, and differences were determined with DeLongs test

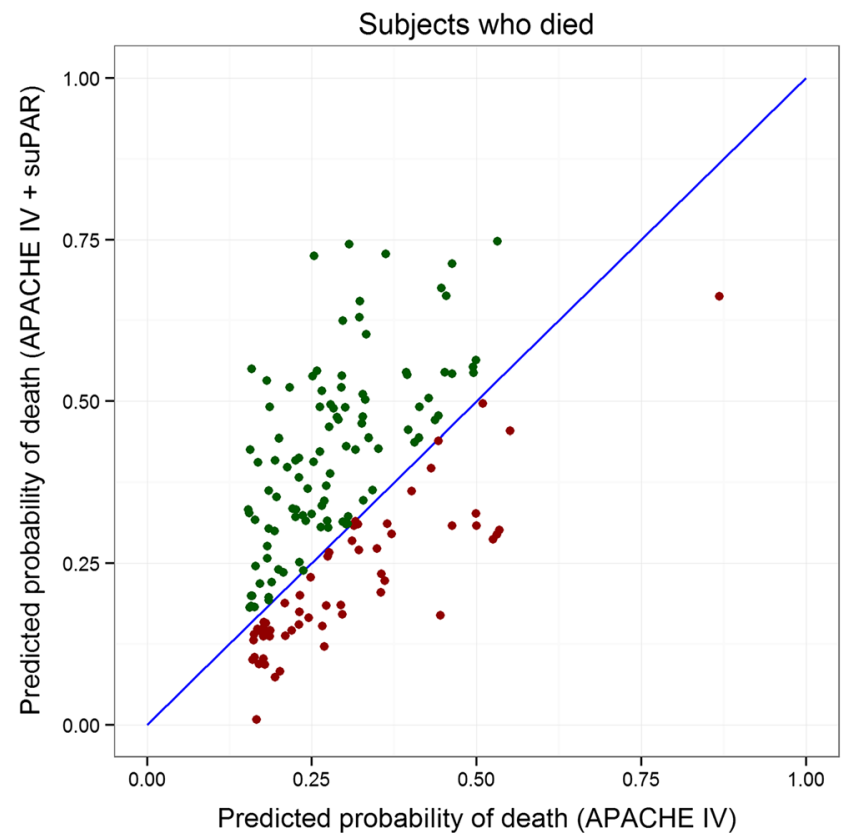

Fig. 2 Risk reclassification for APACHE IV and suPAR combined. The $x$-axis represents the predicted probability of death for the model using only the APACHE IV score, the $y$-axis for the model with APACHE IV and suPAR combined. The blue line represents no difference in predicted probability, the greens dots subjects with

biological markers or clinical disease severity scores to improve mortality prediction. Combining biochemical and clinical parameters has previously improved outcome prediction in patients with lung injury [26]. There was no increase in the ROC-AUC when combining suPAR with the APACHE IV score, SOFA score, oxygenation index, or Berlin classification. However,
$O R$ odds ratio, $S U P A R$ soluble urokinase plasminogen activator receptor, $A R D S$ acute respiratory distress syndrome, $R O C$-AUC receiver operator characteristic curve

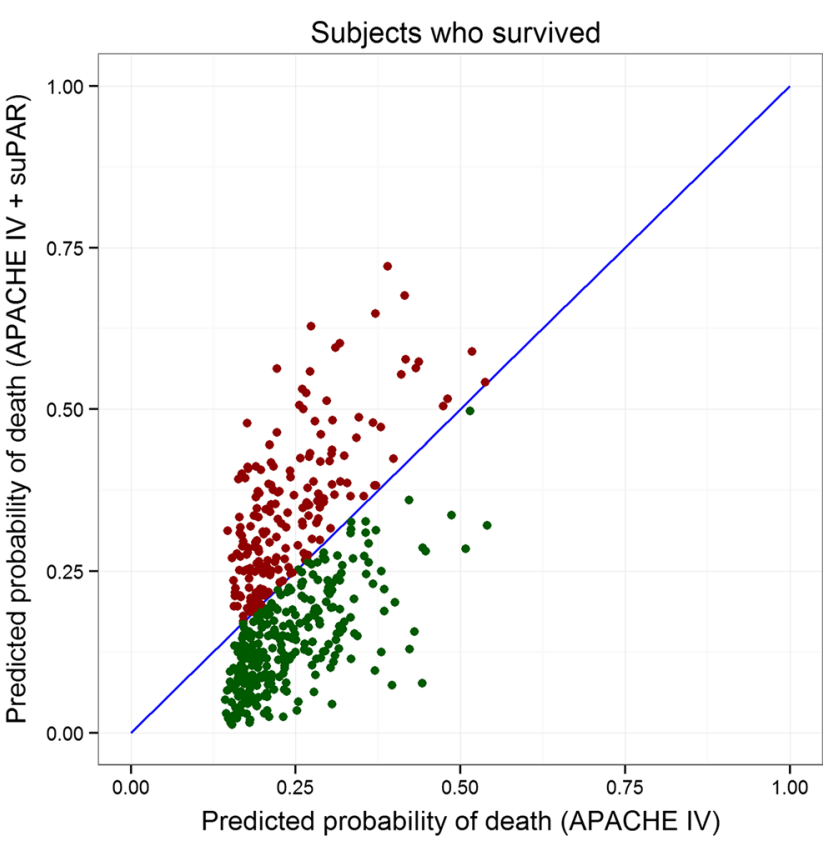

more accurate risk prediction using the APACHE IV plus suPAR model, the red dots those with less accurate risk prediction using the combined model. Subjects that died and subjects that survived were shown in different graphs because the direction of improved probability of the combined model differs for both groups

limitations of the use of ROC-AUCs for determination of the predictive value of biomarkers have been addressed [27]. High odds ratios are needed for an individual biomarker to improve the ROC-AUC [28]. Furthermore discrimination, measured by the ROC-AUC, determines the probability of a case being assigned a higher risk than a control, whereas classification assesses the 
allocation of patients in risk groups based on predicted probability. The net reclassification index (NRI) compares the net reassignment in risk groups of one prediction model to the other [9]. In risk prediction for cardiovascular disease the NRI has been used to show the additional value of adding HDL cholesterol to a prediction model without HDL cholesterol. Although the ROC-AUC did not improve, net reclassification improved by $12 \%$ in the model including HDL cholesterol [9]. In ARDS the NRI has been used to show the value of adding a set of five biomarkers to a clinical prediction score (APACHE III score) [29]. In this study both the ROC-AUC (0.68 vs. 0.75$)$ and the NRI (22\%) improved in the derivation cohort, but in the validation cohort only the NRI showed significant improvement $(17 \%)$ of the model including the biomarkers. In our study combining suPAR with the APACHE IV score, SOFA score, OI, and Berlin classification resulted in remarkable increases in net reclassification indexes, indicating that a substantial number of patients were more accurately classified as survivor or non-survivor. This implies that combining suPAR with existing disease severity scores does provide additional prognostic value.

Our study has several strengths. First, our study involved few inclusion and exclusion criteria, mimicking the clinical setting in which biological markers would be used, ensuring the clinical relevance of the results. Second, the influence of disease severity, admission type, and cause of ARDS was thoroughly investigated. Third, dedicated researchers scored the presence of ARDS, ensuring that no cases were missed. Fourth, the researchers were trained in the use of the ARDS criteria stated by the AECC, in order to attain correct classification of ARDS severity.

Our study also has weaknesses. First, there was a relative underrepresentation of mild ARDS cases in our cohort. This could be due to the tertiary care nature of both participating hospitals, leading to a relatively high overall mortality in this cohort. The high mortality might have contributed to the low prognostic value of suPAR in this cohort. Second, only plasma suPAR was measured. Since suPAR is produced locally in the lungs of ARDS patients, comparing the prognostic value and bioavailability of suPAR in plasma and bronchoalveolar lavage fluid would have been insightful. The design of this study, however, did not allow bronchoalveolar lavage sampling. Third, it could be argued that the measurement of suPAR at additional time points during admission may yield greater insight into identifying recovering vs. non-recovering patients. However insightful, additional measurement of suPAR over time did not fit within the design of this study, aimed at maximum relevancy for use in clinical practice. Furthermore previous research has demonstrated suPAR levels to be stably elevated during the entire first week on the ICU [6]. Finally, combining suPAR with clinical severity scores did not improve the ROC-AUC, yet many other biological markers have been reported as possibly useful in the prediction of mortality in ARDS. Combining suPAR with other biomarkers instead of disease severity scores might have led to improvement of prediction of ICU mortality.

A potential role for suPAR in ICU mortality prediction models may be suggested because suPAR improved the net reclassification when it was added to existing severity scores. However, we do not recommend collection of suPAR in clinical settings at the present moment. The predictive value of combinations of biomarkers for mortality in ARDS patients needs to be confirmed, and possibly improved, before clinical implementation can be initiated. Individual patients can benefit from improved prediction of mortality in two ways: high-risk patients may require aggressive interventions early on, whereas very low-risk patients may profit from withholding possibly dangerous therapies.

\section{Conclusion}

Higher levels of suPAR were found with increasing severity of ARDS, and among those who died compared to survivors. Furthermore, increasing levels of suPAR were associated with less ventilator-free days and ICUfree days. The prognostic value for death of plasma levels of suPAR, however, was low. Nevertheless, suPAR increased the net reclassification index when combined with other predictors of mortality.

Acknowledgments ViroGates A/S, Denmark, donated the ELISA kits for measuring suPAR free of charge. The company had no influence on study design, results, and the decision to publish results. Supported by the Center for Translational Molecular Medicine (http://www.ctmm.nl), project MARS (grant 04I-201).

Conflicts of interest MJS serves as an advisor of Virogates A/S, Denmark. He has no financial interests in the company. The authors declare that they have no competing interests. 


\section{References}

1. Esteban A, Frutos-Vivar F, Muriel A, Ferguson ND, Peñuelas O, Abraira V et al (2013) Evolution of mortality over time in patients receiving mechanical ventilation. Am J Respir Crit Care Med 188(2):220

2. Erickson SE, Martin GS, Davis JL, Matthay MA, Eisner MD, NIH NHLBI ARDS Network (2009) Recent trends in acute lung injury mortality: 1996-2005. Crit Care Med 37(5):1574

3. Barnett N, Ware LB (2011) Biomarkers in acute lung injury-marking forward progress. Crit Care Clin 27(3):661-683

4. Sapru A, Calfdee CS, Liu KD, Kangelaris K, Hansen H, Pawlikowska L, Ware LB, Alkhouli MF, Abbot J, Matthay MA, The NHLBI ARDS Network (2015) Plasma soluble thrombomodulin levels are associated with mortality in the acute respiratory distress syndrome. Intensive Care Med 41:470-478

5. Thun $\varnothing$ M, Machoa B, Eugen-Olsen J (2009) suPAR: the molecular crystal ball. Dis Markers 27:157-172

6. Koch A, Voigt S, Kruschinski C, Sanson E, Dückers H, Horn A, Yagmur E, Zimmermann H, Trautwein C, Tacke F (2011) Circulating soluble urokinase plasminogen activator receptor is stably elevated during the first week of treatment in the intensive care unit and predicts mortality in critically ill patients. Crit Care 15:R63

7. Jalkanen V, Yang R, Linko R, Huhtala H, Okkonen M, Varpula T, Pettila V, Tenhunen J, The FINNALI Study Group (2013) SuPAR and PAI-1 in critically ill, mechanically ventilated patients. Intensive Care Med 39:489-496

8. Suberviola B, Castellanos-Ortega A, Ruiz AR, Lopez-Hoyos M, Santibanez M (2013) Hospital mortality prognostication in sepsis using the new biomarkers suPAR and proADM in a single determination on ICU admission. Intensive Care Med 39:1945-1952

9. Pencina MJ, D’Agostino RB Sr, D'Agostino RB Jr, Vasan RS (2008) Evaluating the added predictive ability of a new marker: from area under the ROC curve to reclassification and beyond. Statist Med 27:157-172
10. Klein Klouwenberg PM, Ong DS, Bos LD, de Beer FM, van Hooijdonk RT, Huson MA, Straat M, van Vught LA, Wieske L, Horn J, Schultz MJ, van der Poll T, Bonten MJM, Cremer OL (2013) Interobserver agreement of Centers for Disease Control and Prevention criteria for classifying infections in critically ill patients. Crit Care Med 41(10):2373-2378

11. Slutsky S, Ranieri VM (2013) Ventilator-induced lung injury. N Engl J Med 369:2126-2136

12. The National Heart, Lung, and Blood Institute Acute Respiratory Distress Syndrome (ARDS) Clinical Trials Network (2006) Comparison of two fluid-management strategies in acute lung injury. N Engl J Med 354:2564-2575

13. Dellinger R, Levy M, Carlet J, Bion J, Parker M, Jaeschke R, Reinhart K, Angus D, Brun-Buisson C, Beale R, Calandra T, Dhainaut J, Gerlach H, Harvey M, Marini J, Marshall J, Ranieri M, Ramsay G, Sevransky J, Thompson B, Townsend S, Vender J, Zimmerman J, Vincent J (2008) Surviving Sepsis Campaign: international guidelines for management of severe sepsis and septic shock: 2008. Crit Care Med 36(1):296-327

14. Bernard G, Artigas A, Brigham K, Carlet J, Falke K, Hudson L, Lamy M, LeGall J, Morris A, Spragg R (1994) Report of the American-European consensus conference on acute respiratory distress syndrome: definitions, mechanisms, relevant outcomes, and clinical trial coordination. Consensus Committee. J Crit Care 9(1):72-81

15. ARDS Definition Task Force et al (2012) Acute respiratory distress syndrome: the Berlin definition. JAMA 307(23):2526-2533

16. Ferguson N, Fan E, Camporota L, Antonelli M, Anzueto A, Beale R, Brochard L, Brower R, Esteban A, Gattinoni L, Rhodes A, Slutsky A, Vincent J, Rubenfeld G, Thompson B, Ranieri V (2012) The Berlin definition of ARDS: an expanded rationale, justification, and supplementary material. Intensive Care Med 38(10):1573-1582
17. Zimmerman JE, Kramer AA, McNair DS, Malila FM (2006) Acute Physiology and Chronic Health Evaluation (APACHE) IV: hospital mortality assessment for today's critically ill patients. Crit Care Med 34(5): 1297

18. Vincent JL, de Mendonça A, Cantraine F, Moreno R, Takala J, Suter PM, Sprung CL, Colardyn F, Blecher S (1998) Use of the SOFA score to assess the incidence of organ dysfunction/failure in intensive care units: results of a multicenter, prospective study. Working group on "sepsis-related problems" of the European Society of Intensive Care Medicine. Crit Care Med 26(11):1793-1800

19. Trachsel D, McCrindle BW, Nakagawa S, Bohn D (2005) Oxygenation index predicts outcome in children with acute hypoxemic respiratory failure. Am J Respir Crit Care Med 172:206-211

20. Robin X, Turck N, Hainard A, Tiberti $\mathrm{N}$, Lisacek F, Sanchez JC, Müller M (2011) pROC: an open-source package for $\mathrm{R}$ and $\mathrm{S}+$ to analyze and compare ROC curves. BMC Bioinformatics $12: 77$

21. R Core Team (2015) R: A language and environment for statistical computing. R Foundation for Statistical Computing, Vienna, Austria.

http://www.R-project.org

22. Plewes K, Royakkers AA, Hanson J, Hasan MM, Alam S, Ghose A, Maude RJ, Stassen PM, Charunwatthana P, Lee SJ, Turner GD, Dondorp AM, Schultz MJ (2014) Correlation of biomarkers for parasite burden and immune activation with acute kidney injury in severe falciparum malaria. Malaria $\mathbf{J}$ 13:91

23. Backes Y, van der Sluijs KF, Tuip de Boer AM, Hofstra JJ, Vlaar AP, Determann RM, Knape P, Mackie DP, Schultz MJ (2011) Soluble urokinasetype plasminogen activator receptor levels in patients with burn injuries and inhalation trauma requiring mechanical ventilation: an observational cohort study. Crit Care 15(6):R270

24. Vincent JL, Opal SM, Marshall JC (2010) Ten reasons why we should NOT use severity scores as entry criteria for clinical trials or in our treatment decisions. Crit Care Med 38(1):283-287 
25. Quach S, Hennessy DA, Faris P, Fong A, Quan H, Doig C (2009) A comparison between the APACHE II and Charlson Index Score for predicting hospital mortality in critically ill patients. BMC Health Serv Res 30(9):129

26. Ware LB, Koyama T, Billheimer DD, Wu W, Bernard GR, Thompson BT, Brower RG, Standiford TJ, Martin TR, Matthay MA, NHLBI ARDS Clinical Trials Network (2010) Prognostic and pathogenetic value of combining clinical and biochemical indices in patients with acute lung injury. Chest 137(2):288-296
27. Cook NR (2007) Use and misuse of the receiver operating characteristic curve in risk prediction. Circulation 115(7):928-935

28. Pepe MS, Janes H, Longton G, Leisenring W, Newcomb P (2004) Limitations of the odds ratio in gauging the performance of a diagnostic, prognostic, or screening marker. Am J Epidemiol 159:882-890
29. Calfee CS, Ware LB, Glidden DV, Eisner MD, Parsons PE, Thompson TB, Matthay MA, National Heart, Blood, and Lung Institute Acute Respiratory Distress Syndrome Network (2011) Use of risk reclassification with multiple biomarkers improves mortality prediction in acute lung injury. Crit Care Med 39:711-717 